\title{
Damage Identification in Skeletal Structures using the Virtual Distortion Method in Frequency Domain
}

\author{
Andrzej Świercz, Przemysław Kołakowski, Jan Holnicki-Szulc \\ Smart-Tech Centre \\ Institute of Fundamental Technological Research \\ Świetokrzyska 21, 00-049 Warsaw, Poland \\ http://smart.ippt.gov.pl/
}

\begin{abstract}
The paper is a continuation of research on the application of a structural reanalysis method - the Virtual Distortion Method - to Structural Health Monitoring. The first approach, formulated previously in the time domain, suffered from a considerable numerical cost. In order to reduce it, this paper presents an alternative approach, formulated in the frequency domain thanks to the assumption of using harmonic excitation (quasi-static problem). The hardware devices supposed to collect structural responses are piezoelectric patch sensors. The software tool for damage identification solves a nonlinear least squares optimization problem by employing analytically derived sensitivities. Strains are the analysed quantities, contributing to the objective function. Considerations are restricted to skeletal structures and a simplified dynamic problem with no damping. Effectiveness of the frequency-domain identification is demonstrated in numerical examples. Experimental verification is envisaged.
\end{abstract}

Keywords: damage identification, inverse problem, frequency domain, skeletal structures, reanalysis methods

\section{Introduction}

Vibration-based (low-frequency) Structural Health Monitoring (SHM) has gained a lot of researchers' attention in recent years. The main stream is concentrated on utilizing ambient excitation and applying Operational Modal Analysis [1] to localize and quantify damage. The environmental type of excitation has its weak points however, therefore the newest trend is to enrich the OMA with eXogenous input (OMAX) of known characteristic to facilitate damage detection [2]. Degradation of stiffness is usually examined in SHM and damage-sensitive parameters (e.g. curvature [3], energy [4]) are defined to track stiffness changes in structures. Beside the modal parameters, the antiresonance frequencies [5] may successfully contribute to damage detection as well. Defining indicators able to distinguish between the actual damage and the influence of environmental factors (e.g. temperature) on structural response is a challenging research topic [6]. A number of effective methods for system identification and subsequent vibration-based SHM have been elaborated e.g. Eigensystem Realization Algorithm [7], Stochastic Subspace 
Identification [8], Complex Mode Indicator Function [9], PolyMAX [10]. Some of them, e.g. Least Squares Complex Exponential [11], have already become standards in industry applications.

This paper continues to present the capabilities of a structural reanalysis method the Virtual Distortion Method (VDM) - in application to SHM. The VDM is a method of structural reanalysis, which consists in imposing appropriate initial strains in the structure in order to model required modifications of structural parameters in an efficient way. An overview of VDM applications with short characteristics of other reanlysis methods, e.g. Combined Approximations [12], can be found in [13]. The distinctive features of the VDM are:

- exact (not approximate) formulation, capturing all system's features in the influence matrix

- ability to handle non-linear (piece-wise linear) constitutive law

- dynamic reanalysis in the time or frequency domains, enabling modification of both stiffness and inertia parameters

The VDM-based time domain approach to SHM, proposed in [14], linked the software tool for solving the inverse problem of identification via gradient-based optimization with the hardware concept of utilizing piezoelectric patch sensors to collect structural responses due to impulse excitation. The formulation of the VDM-based piezodiagnostics in the time domain was general enough to handle an impulse excitation signal and required only a few sensors for analysis. However it suffered from a considerable computational cost. This fact spurred the development of an alternative approach, presented in this paper. The idea is to transfer the problem to the frequency domain thanks to the assumption of applying harmonic excitation, thus replacing the dynamic analysis with the quasi-static one. The harmonic excitation is inherent in some vibroacoustic problems, e.g. rotating machinery, or can be applied to real structures by shakers. Apart from the in-situ applications, it is also commonly used in laboratory experiments.

The main point of this paper is to present a VDM-based methodology of damage identification (location and intensity) for skeletal structures (trusses and beams) by considering not only stiffness but also mass modifications. It is assumed that the system does not vary in time during measurements. A simplified dynamic problem with no damping is considered. Consequently, resonance frequencies are avoided in analysis. Analytically derived sensitivities are effectively utilized in an optimization algorithm leading to damage identification thanks to solving an inverse problem. Piezoelectric sensors are assumed to capture structural response to a low-frequency (up to $1 \mathrm{kHz}$ ) harmonic excitation. Experimental verification of the proposed approach has been just announced.

\section{Parameter modification by the Virtual Distortion Method}

\subsection{General characteristic of VDM}

The Virtual Distortion Method (VDM)[15] is conceptually similar to the initial strains approach. Introduction of initial strains in structures was primarily proposed to model plasticity. However, the local imposition of an initial strain leads to violation of equilibrium conditions and the solution proceeds in iterations. On the contrary, the VDM 
approach is able to produce such solution in one step thanks to defining all local-global interrelations in a structure in advance. The collection of all the local-global responses, including information about structural topology, materials and boundary conditions, is called the influence matrix within the framework of VDM. This matrix makes an essential difference between the VDM and initial strains approach.

The VDM belongs to fast reanalysis methods in structural mechanics. This means that an initial FEM response is necessary for introducing further modification by determining proper fields of virtual distortions. A comparative review of reanalysis methods can be found in [16], where equivalence between the VDM and Sherman-Morrison-Woodbury formulas is proved.

The VDM has been used in various problems of structural design (e.g. prestress), optimization (e.g. topology remodelling) and control (e.g. damping of vibrations) thus far. It has also turned out to be a promising tool for SHM. The first attempt to handle the problem of damage identification using the VDM was made during the project PiezoDiagnostics [17]. Low-frequency impulse excitation (e.g. windowed sine pulse) was applied and time responses of a structure were captured by piezoelectric transducers. The electrical responses (voltage) of the transducers were proportional to mechanical strains. The first formulation assumed only stiffness degradation as a damage modelling parameter. The extension of the VDM remodelling approach, including mass modifications in the time domain, was described in [13], in which a review of all VDM applications to date can be found.

\subsection{Influence matrix}

The paper is concerned with skeletal structures. In further description let us assume that the lowercase indices, e.g. $i$, refer to elements in local co-ordinates and the uppercase ones, e.g. $M$, to nodes in global co-ordinates. Einstein's summation convention has been adopted. Underlined indices are exempt from summation.

Let us first demonstrate the concept of the influence matrix for truss structures in static analysis. Each component of the influence matrix $D_{i j}^{\varepsilon}$ describes strains in the truss member $i$ caused by the unit virtual distortion $\varepsilon_{j}^{0}=1$ (unit axial tensile strain for trusses) applied to the member $j$. The unit virtual distortion is imposed in numerical calculations as a pair of self-equilibrated compensative forces of reverse signs (equivalent to a unit strain) applied to the nodes of the strained element. The influence matrix $D_{i j}^{\varepsilon}$ collects $n$ influence vectors, where $n$ denotes the number of truss elements. In order to build an influence vector, a solution of a standard linear elastic problem by the Finite Element Method has to be found:

$$
K_{M N} u_{N}=f_{M}
$$

with $K_{M N}$ being the stiffness matrix, $u_{N}$ - displacement vector and $f_{M}$ - force vector in global co-ordinates. Usually, the obtained global displacements serve to calculate a corresponding response in local strains:

$$
\varepsilon_{i}=G_{i K} u_{K}
$$

with $G_{i K}$ being the geometric matrix, which transforms global degrees of freedom to local strains. The response in strains is most often considered for building an influence vector. However, storage of any other required response, i.e., displacements, stresses or forces, is also useful. 
The external force vector $f$ in (1) corresponds to two compensative forces (axial tensile forces in case of truss structures) applied to a structural member, equivalent with application of a unit strain to the unconstrained member (see the diagonal element in Fig. 1, taken out of structure, subjected to a pair of forces). The response of the structure to the imposition of the unit virtual distortion $\varepsilon_{5}^{0}=1$ is depicted by the deformed configuration in Fig. 1.

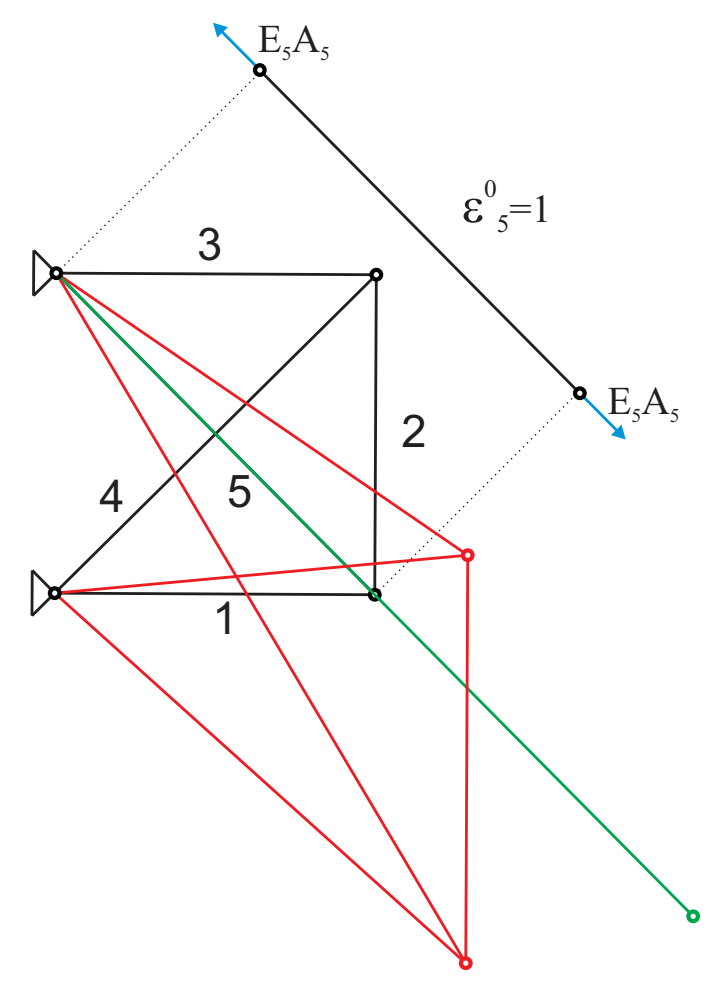

Figure 1: Deformed structural configuration as a response to a static virtual distortion.

Thus to build the influence matrix $D_{i j}^{\varepsilon}, n$ solutions of a linear elastic problem have to be found. The set (1) has to be solved with $n$ different right-hand sides corresponding to $n$ pairs of compensative forces applied successively in each structural member. This way, the influence matrix stores information about the entire structure properties including topology, material characteristics and boundary conditions in calculation of structural response.

Note that the static influence matrix for statically determinate structures becomes identity (zero redundancy means no inter-relations between members) and the VDM loses its major tool (only in statics).

Analogously, the influence matrix can be built in dynamic analysis, where the response due to impact load in the first time step is collected. Integration of the equations of motion is performed by the Newmark algorithm over some predefined period of time. In order to build the influence matrix in dynamics, a pair of self-equilibrated forces equivalent to unit strain (analogously to statics) is applied to a truss member in the first time step only. Such a perturbation introduced to the structure is called an impulse virtual distortion (see Fig. 2). In subsequent time steps, the influence of the distortion on the structure is examined over the discretized time period. Structural response in each time step corresponds to an influence vector in statics, so the collection of such responses from all time steps provides an influence "vector" in dynamics. This vector has an extra dimension, which is time. An assembly of dynamic influence "vectors" constitute the dynamic influence 
"matrix", which has two dimensions corresponding to the number of structural members (like in statics) and the third dimension - time. Generally, the dynamic influence matrix is time dependent, however for harmonic excitation, it becomes quasi static, because only amplitudes of responses are stored. The influence matrix for harmonic excitation, further considered in the paper, does not depend upon time, however it does depend upon the frequency of excitation.

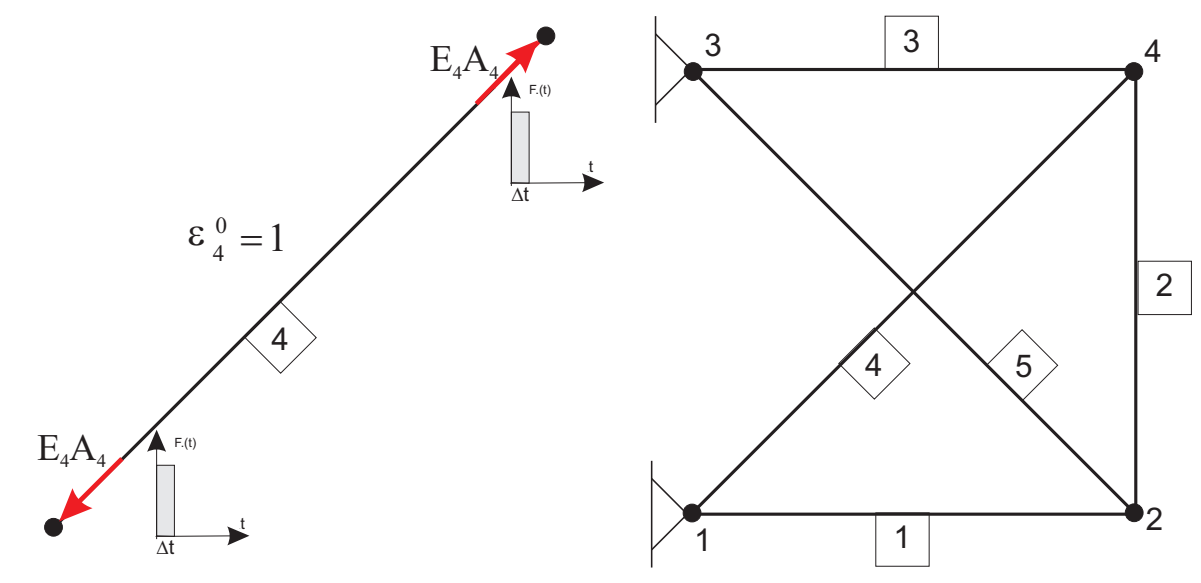

Figure 2: Impulse virtual distortion applied in an element.

\subsection{Stiffness modelling in truss elements}

Let us confine our considerations to truss structures in the elastic range first. Let us consider introducing a field of initial strains $\varepsilon_{j}^{0}$ (called virtual distortions) into a truss structure. This action will induce residual displacements and strains in the structure, expressed as follows (cf. [15]):

$$
\begin{aligned}
u_{K}^{\mathrm{R}} & =B_{K j}^{\varepsilon} \varepsilon_{j}^{0} \\
\varepsilon_{i}^{\mathrm{R}} & =D_{i j}^{\varepsilon} \varepsilon_{j}^{0}
\end{aligned}
$$

The matrix $B_{K j}^{\varepsilon}$ collects structural responses in displacements to the unit virtual distortion $\varepsilon_{j}^{0}=1$ equivalent with the unit strain. The matrix $D_{i j}^{\varepsilon}$ collects corresponding structural responses in strains. Both matrices are marked with the upper subscript $\varepsilon$, which indicates the fact they were built due to imposition of strain-like virtual distortions. The two influence matrices are related by the geometric matrix $G_{i K}(\mathrm{cf} .(2))$ as follows:

$$
D_{i j}^{\varepsilon}=G_{i K} B_{K j}^{\varepsilon}
$$

Residual stresses are expressed by:

$$
\sigma_{i}^{\mathrm{R}}=E_{\underline{i}}\left(D_{\underline{i j}}^{\varepsilon}-\delta_{\underline{i j}}\right) \varepsilon_{j}^{0}
$$

where $E_{i}$ denotes the Young's modulus and $\delta_{i j}$ - the Kronecker's delta.

Assume that application of external load to the structure provokes linear elastic response $u_{K}^{\mathrm{L}}, \varepsilon_{i}^{\mathrm{L}}, \sigma_{i}^{\mathrm{L}}$, which will be superposed with the residual response $u_{K}^{\mathrm{R}}, \varepsilon_{i}^{\mathrm{R}}, \sigma_{i}^{\mathrm{R}}$. Thus in view of $(3),(4),(6)$ we get:

$$
\begin{aligned}
u_{K} & =u_{K}^{\mathrm{L}}+u_{K}^{\mathrm{R}} \\
\varepsilon_{i} & =\varepsilon_{i}^{\mathrm{L}}+\varepsilon_{i}^{\mathrm{R}} \\
\sigma_{i} & =\sigma_{i}^{\mathrm{L}}+\sigma_{i}^{\mathrm{R}}=E_{\underline{i}}\left(\varepsilon_{\underline{i}}-\varepsilon_{\underline{i}}^{0}\right)
\end{aligned}
$$


Relation between element forces $p_{i}$ and stresses $\sigma_{i}$ is known via the cross-sectional areas $A_{i}$ :

$$
p_{i}=A_{\underline{i}} \sigma_{\underline{i}}
$$

Let us now take into account structural geometry modifications exemplified by changes of cross-sectional area of a member. This means considering of a modified value $\hat{A}_{i}$. In view of (9) and (10), we can express element forces in the original structure with introduced virtual distortion field (called distorted structure) and in the modified structure, as follows:

$$
\begin{aligned}
& p_{i}=E_{\underline{i}} A_{\underline{i}}\left(\varepsilon_{\underline{i}}-\varepsilon_{\underline{i}}^{0}\right) \\
& \hat{p}_{i}=E_{\underline{i}} \hat{A}_{\underline{i}} \hat{\varepsilon}_{\underline{i}}
\end{aligned}
$$

The main postulate of the VDM in static remodelling requires that local strains (including plastic strains) and forces in the distorted and modified structure are equal:

$$
\begin{aligned}
\varepsilon_{i} & =\hat{\varepsilon}_{i} \\
p_{i} & =\hat{p}_{i}
\end{aligned}
$$

This postulate leads to the following relation:

$$
E_{\underline{i}} A_{\underline{i}}\left(\varepsilon_{\underline{i}}-\varepsilon_{\underline{i}}^{0}\right)=E_{\underline{i}} \hat{A}_{\underline{i}} \varepsilon_{\underline{i}}
$$

Equation (15) provides the coefficient of the stiffness change $\mu_{i}$ for each truss element $i$ as the ratio of the modified parameter $\hat{A}_{i}$ to the initial one $A_{i}$ :

$$
\mu_{i}=\frac{\hat{A}_{\underline{i}}}{A_{\underline{i}}}=\frac{\varepsilon_{\underline{i}}-\varepsilon_{\underline{i}}^{0}}{\varepsilon_{\underline{i}}}
$$

Note that the coefficient $\mu_{i}$ may be equivalently expressed as the ratio of the initial to modified Young's modulus of a truss element. If $\mu_{i}=1$ we deal with an intact structure. Variation of the coefficient in the range $0 \leq \mu_{i} \leq 1$ means degradation of stiffness and in the range $\mu_{i} \geq 1$ increase of stiffness. Substituting (4), (8) into (16) we get a set of equations for $\varepsilon_{j}^{0}$, which must be solved for an arbitrary number of modified elements (usually small compared to all elements in the structure), described by a coefficient $\mu_{i}$ different than 1:

$$
\left[\delta_{\underline{i j}}-\left(1-\mu_{\underline{i}}\right) D_{\underline{i j}}^{\varepsilon}\right] \varepsilon_{j}^{0}=\left(1-\mu_{\underline{i}}\right) \varepsilon_{\underline{i}}^{\mathrm{L}}
$$

In dynamics, a residual response is a discrete convolution of the influence matrix and virtual distortions. The time-dependent residual displacement, strain and stress vectors can be expressed as follows (cf. (3), (4), (6)):

$$
\begin{aligned}
u_{K}^{\mathrm{R}}(t) & =\sum_{\tau=0}^{t} B_{K j}^{\varepsilon}(t-\tau) \varepsilon_{j}^{0}(\tau) \\
\varepsilon_{i}^{\mathrm{R}}(t) & =\sum_{\tau=0}^{t} D_{i j}^{\varepsilon}(t-\tau) \varepsilon_{j}^{0}(\tau) \\
\sigma_{i}^{\mathrm{R}}(t) & =E_{\underline{i}}\left(\sum_{\tau=0}^{t-1} D_{\underline{i j}}^{\varepsilon}(t-\tau)+D_{\underline{i j}}^{\varepsilon}(0)-\delta_{\underline{i j}}\right) \varepsilon_{j}^{0}(\tau)
\end{aligned}
$$


All the relations for statics are valid for dynamics as well. While most quantities vary in time, the stiffness change coefficient, defined analogously to (16), remains time-independent:

$$
\mu_{i}=\frac{\hat{A}_{\underline{i}}}{A_{\underline{i}}}=\frac{\varepsilon_{\underline{i}}(t)-\varepsilon_{\underline{i}}^{0}(t)}{\varepsilon_{\underline{i}}(t)}
$$

The set of equations to be solved for distortions in dynamics looks analogously to (17):

$$
\left[\delta_{\underline{i j}}-\left(1-\mu_{\underline{i}}\right) D_{\underline{i j}}^{\varepsilon}(0)\right] \varepsilon_{j}^{0}(t)=\left(1-\mu_{\underline{i}}\right) \varepsilon_{\underline{i}}^{\neq t}(t)
$$

where $\varepsilon_{i}^{\neq t}(t)$ denotes strains cumulated before the current step $t$ :

$$
\varepsilon_{i}^{\neq t}(t)=\varepsilon_{i}^{\mathrm{L}}(t)+\sum_{\tau=0}^{t-1} D_{i j}^{\varepsilon}(t-\tau) \varepsilon_{j}^{0}(\tau)
$$

Note that the matrix on the left-hand side of (22) is constant in time. Only the right-hand side vector of (22) has to be modified in every time step, according to (23). Similarly to (17), the set (22) may be local if structural remodelling is performed. In identification problems however, in which the location of a damaged/modified member is sought, the set (22) concerns all elements potentially changed (usually the whole structure).

\subsection{Mass modelling at truss nodes}

In dynamic analysis, virtual distortions modelling stiffness changes, considered in section 2.3, are not sufficient to describe structural behavior completely. There is a need to introduce another perturbation, able to model inertia changes. To this end, a single, unequilibrated unit force is applied to a node of the structure in directions corresponding to degrees of freedom (see Fig. 3).

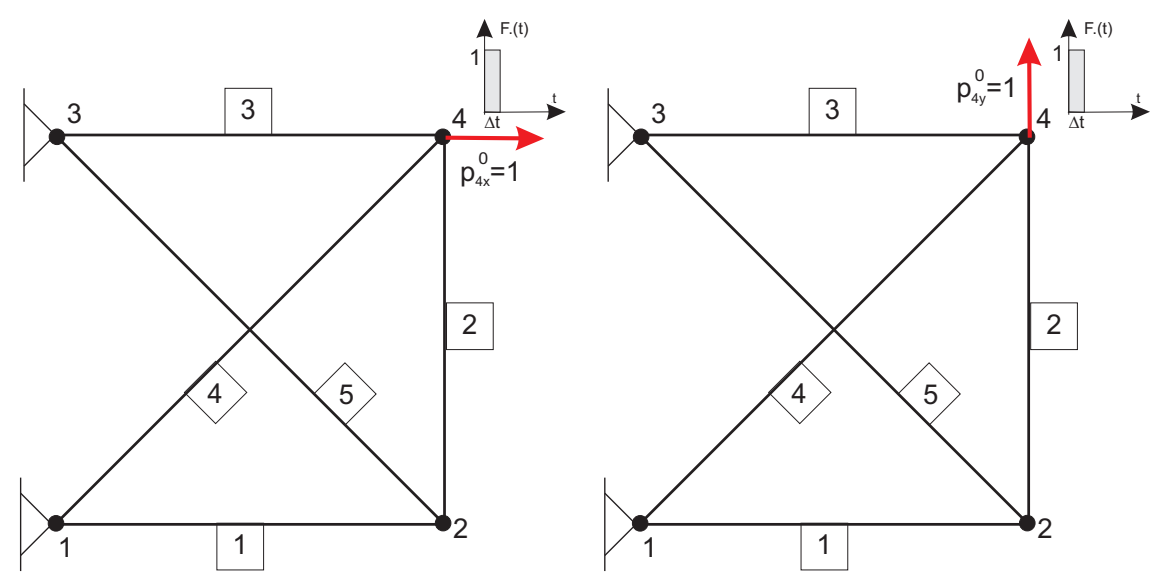

Figure 3: Impulse force distortion applied in the direction of DOF at a node.

The unbalanced force is applied only in the first time step, so this kind of perturbation is called an impulse force distortion $f_{L}^{0}$. Consequently, a relevant influence matrix $B_{K L}^{\mathrm{f}}$, collecting response in displacements to impulse force distortions, is built. Thus, in case of inertia changes, the residual displacements and strains (cf. (18), (19)) are expressed as:

$$
\begin{aligned}
& u_{K}^{\mathrm{R}}(t)=\sum_{\tau=0}^{t} B_{K L}^{\mathrm{f}}(t-\tau) f_{L}^{0}(\tau) \\
& \varepsilon_{i}^{\mathrm{R}}(t)=\sum_{\tau=0}^{t} D_{i L}^{\mathrm{f}}(t-\tau) f_{L}^{0}(\tau)
\end{aligned}
$$


where the relation between matrices $D_{i L}^{\mathrm{f}}$ and $B_{K L}^{\mathrm{f}}$ is analogous to (5):

$$
D_{i L}^{\mathrm{f}}=G_{i K} B_{K L}^{\mathrm{f}}
$$

Differentiating (24) twice with respect to time gives:

$$
\ddot{u}_{K}^{\mathrm{R}}(t)=\sum_{\tau=0}^{t} \ddot{B}_{K L}^{\mathrm{f}}(t-\tau) f_{L}^{0}(\tau)
$$

Let us now take into account structural mass modifications accompanying the changes of cross-sectional area of a member. We can express simplified equations of motion for the distorted and modified structures as follows:

$$
\begin{aligned}
& M_{K L} \ddot{u}_{L}(t)+K_{K L} u_{L}(t)=f_{K}(t)+f_{K}^{0}(t) \\
& \hat{M}_{K L} \hat{\ddot{u}}_{L}(t)+K_{K L} u_{L}(t)=\hat{f}_{K}(t)
\end{aligned}
$$

The main postulate of the VDM in dynamic remodelling says that inertia forces and accelerations in the distorted and modified structure are equal. Taking this into account and subtracting (28) from (29), the relation for determination of impulse force distortions is obtained:

$$
\Delta M_{K L} \ddot{u}_{L}(t)+f_{K}^{0}(t)=0
$$

where $\Delta M_{K L}$ determines a modification to the global mass matrix:

$$
\Delta M_{K L}=\hat{M}_{K L}-M_{K L}=\sum_{i}\left(\mu_{i}-1\right) M_{K L}^{i}
$$

in which the matrix $M^{i}$ is the consistent mass matrix for element $i$ after transformation to the global coordinate system. The summation in (31) denotes aggregation over all finite elements $i$. Sometimes, the aggregation does not have to be performed. Each component of $\Delta M$ (corresponding to an element) is stored separately, which enables to account only for the locations subject to a change. This is important in remodelling the design, but not the case of an identification problem in which modified location are to be found.

Substituting (27) into (30) and rearranging, the following set of equations is obtained, with the unknown vector $f_{L}^{0}$, which models mass changes:

$$
\left[\delta_{K L}+\Delta M_{K M} \ddot{B}_{M L}^{\mathrm{f}}(0)\right] f_{L}^{0}(t)=-\Delta M_{K M} \ddot{u}_{M}^{\neq t}(t)
$$

where $\ddot{u}_{M}^{\neq t}(t)$ denotes strains cumulated before the current step $t$ :

$$
\ddot{u}_{M}^{\neq t}(t)=\ddot{u}_{M}^{\mathrm{L}}(t)+\sum_{\tau=0}^{t-1} \ddot{B}_{M L}^{\mathrm{f}}(t-\tau) f_{L}^{0}(\tau)
$$

Note that the set (32) is local (only modified locations are considered) in case of design remodelling and global in case of parameter identification (all locations are potentially subject to a change). 

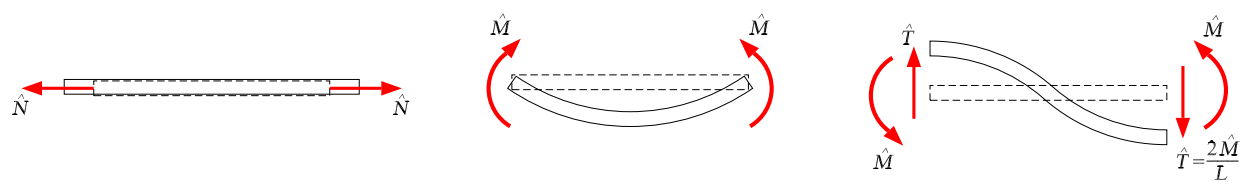

Figure 4: Virtual distortion states for a beam element.

\subsection{Modifications in beams}

For a 2D beam element there are 3 components of virtual distortions that have to be applied. The 3 distortion components correspond to 3 states of deformation (see Fig. 4) in orthogonal base, obtained through the solution of the eigenproblem of the $2 \mathrm{D}$ beam element stiffness matrix. So apart from the axial type of distortion $\varepsilon_{i}$ (as for trusses), the beam distortions also include pure bending $\kappa_{i}$ and bending plus shear $\chi_{i}$ terms.

Further in the paper if a Latin lowercase index describes a quantity for a beam element, one should remember that it refers to a triplet of the above-described distortions (not a single axial strain). Thus, for the $2 \mathrm{D}$ beam model, apart from the modification coefficient corresponding to axial strain (cf. (21)), also analogous coefficients for bending (the ratio of the modified $\hat{J}_{i}$ to initial $J_{i}$ moment of inertia) may be defined:

$$
\begin{aligned}
& \mu_{i}^{\varepsilon}=\mu_{i}^{\mathrm{A}}=\frac{\hat{A}_{\underline{i}}}{A_{\underline{i}}}=\frac{\varepsilon_{\underline{i}}(t)-\varepsilon_{\underline{i}}^{0}(t)}{\varepsilon_{\underline{i}}(t)} \\
& \mu_{i}^{\kappa}=\mu_{i}^{\mathrm{J}}=\frac{\hat{J}_{\underline{i}}}{J_{\underline{i}}}=\frac{\kappa_{\underline{i}}(t)-\kappa_{\underline{i}}^{0}(t)}{\kappa_{\underline{i}}(t)} \\
& \mu_{i}^{\chi}=\mu_{i}^{\mathrm{J}}=\frac{\hat{J}_{\underline{i}}}{J_{\underline{i}}}=\frac{\chi_{\underline{i}}(t)-\chi_{\underline{i}}^{0}(t)}{\chi_{\underline{i}}(t)}
\end{aligned}
$$

As axial response for the 2D beam element is independent from bending/shear, we can practically distinguish two coefficients for analysis i.e. $\mu_{i}^{\varepsilon}$ denoting axial stiffness change and $\mu_{i}^{\kappa}$ denoting bending stiffness change. As a consequence, the consistent mass matrix $6 \times 6$ is divided into two parts - the first one containing only the cross-sectional area $A_{i}$ of a beam and the second one containing only its moment of inertia $J_{i}$. The global mass matrix is assembled as:

$$
M_{K L}=\sum_{i}\left(\stackrel{\mathrm{A}}{M_{K L}^{i}}+\stackrel{\mathrm{J}}{M}_{K L}^{i}\right)
$$

where $i$ denotes a part of the mass matrix in global coordinates, corresponding to the beam element $i$. The increment of mass (cf. (31)) is then expressed as:

$$
\Delta M_{K L}=\hat{M}_{K L}-M_{K L}=\sum_{i}\left(\left(\mu_{i}^{\mathrm{A}}-1\right) \stackrel{\mathrm{A}}{M_{K L}^{i}}+\left(\mu_{i}^{\mathrm{J}}-1\right) M_{K L}^{\mathrm{J}}\right)
$$

\section{Identification problem for harmonic excitation}

\subsection{Problem formulation}

A steady-state problem is characterized by continuous-in-time harmonic excitation of the form:

$$
f(t)=f \sin (\omega t)
$$


where $f, \omega$ denote amplitude and frequency of the exciting force, respectively.

This assumption significantly simplifies considerations of the equation of motion as all mechanical quantities and their derivatives vary in the same harmonic manner e.g. displacements are expressed as:

$$
u(t)=u \sin (\omega t)
$$

Therefore it is quite sufficient to analyze only time-independent amplitude values of the quantities. Consequently, the dynamic problem becomes quasi static, e.g. relations (24), (25) have the same form, but with no time dependency.

As indicated in section 2, we would like to consider both stiffness and mass modifications in the structure e.g. in case of displacements both residual responses (18) and (24) have to be considered. Assuming the two kinds of modifications and taking into account relation (39), the equations of motion for the distorted and modified (with mass and stiffness changes) structures can be expressed as (cf. (28), (29)):

$$
\begin{aligned}
-\omega^{2} M_{K L} u_{L}+K_{K L}\left(u_{L}-u_{L}^{0}\right) & =f_{K}+f_{K}^{0} \\
-\omega^{2} \hat{M}_{K L} \hat{u}_{L}+\hat{K}_{K L} \hat{u}_{L} & =f_{K}
\end{aligned}
$$

where the distortion $u_{L}^{0}$, expressed in global (system) coordinates and introduced solely for the simplicity of notation in (41), corresponds to the stiffness-modelling distortion $\varepsilon_{j}^{0}$, expressed in local (element) coordinates. Subtracting (41) from (42) leads to the relation for $f_{K}^{0}$, similar to $(30)$ :

$$
-\omega^{2} \Delta M_{K L} u_{L}+f_{K}^{0}=0
$$

Note that to obtain (43), the static postulate of VDM (equivalence of element strains and forces - cf. (13) - (15)), expressed in global coordinates, was used:

$$
\begin{aligned}
u_{L} & =\hat{u}_{L} \\
K_{K L}\left(u_{L}-u_{L}^{0}\right) & =\hat{K}_{K L} \hat{u}_{L}
\end{aligned}
$$

When both stiffness and mass changes are analyzed, strains in elements depend on both the distortions $\varepsilon_{j}^{0}$ and $f_{L}^{0}$ :

$$
\varepsilon_{i}=\varepsilon_{i}^{\mathrm{L}}+D_{i j}^{\varepsilon} \varepsilon_{j}^{0}+D_{i L}^{\mathrm{f}} f_{L}^{0}
$$

Analogously, displacements corresponding to global degrees of freedom are expressed by the following relation:

$$
u_{M}=u_{M}^{\mathrm{L}}+B_{M j}^{\varepsilon} \varepsilon_{j}^{0}+B_{M L}^{\mathrm{f}} f_{L}^{0}
$$

Substituting (46) to (16) and (47) to (43), a set of $j+L$ equations is assembled:

$$
\left[\begin{array}{cc}
\delta_{i j}-\left(1-\mu_{\underline{i}}\right) D_{\underline{i j}}^{\varepsilon} & -\left(1-\mu_{\underline{i}}\right) D_{\underline{i} L}^{\mathrm{f}} \\
-\omega^{2} \Delta M_{K M} B_{M j}^{\varepsilon} & \delta_{K L}-\omega^{2} \Delta M_{K M} B_{M L}^{\mathrm{f}}
\end{array}\right]\left[\begin{array}{c}
\varepsilon_{j}^{0} \\
f_{L}^{0}
\end{array}\right]=\left[\begin{array}{c}
\left(1-\mu_{\underline{i}}\right) \varepsilon_{\underline{i}}^{\mathrm{L}} \\
\omega^{2} \Delta M_{K M} u_{M}^{\mathrm{L}}
\end{array}\right]
$$

The set is local i.e. variables $\varepsilon_{j}^{0}$ and $f_{L}^{0}$ are confined to the modified locations only (relevant elements or degrees of freedom). It is solved using an SVD solver. 


\subsection{Optimization issues}

In most approaches to damage identification, the measured quantity is acceleration, because it is relatively easy to obtain. However the raw acceleration signal in time is never used directly - it requires FFT processing to transfer the analysis into the frequency domain. In the proposed approach a different quantity is measured. It is namely strain in time, measured by piezo-transducers, which is then directly used in the VDM time domain approach (cf. [14]). This paper is focused on harmonic excitation thanks to which only frequency-dependent amplitudes of strains are examined. Thus we can speak about the VDM frequency domain approach. One should note that there is no need for an FFT processing of the time signal as performed in standard frequency-domain methods.

As often in the parameter estimation procedures, we pose the identification task as a nonlinear least squares minimization problem with the objective function expressed in strains:

$$
F(\boldsymbol{\mu})=\sum_{n_{\omega}}\left(\frac{\varepsilon_{k}-\varepsilon_{k}^{\mathrm{M}}}{\varepsilon_{k}^{\mathrm{M}}}\right)^{2}
$$

The function (49) collects responses from selected $n_{\omega}$ frequencies of operation and from $k$ sensors, placed in those elements where non-zero strains of high signal-to-noise ratio are measured. The strain $\varepsilon_{k}$ in an arbitrarily selected location $k$ is influenced by virtual distortions $\varepsilon_{i}^{0}$, which may be generated in any element $i$ of the structure (cf. (4), (8)). One should also note that the modification coefficient $\mu_{i}$, quantifying potential damage and used as a variable in optimization, depends upon the virtual distortions $\varepsilon_{i}^{0}$ nonlinearly (cf. (16)). As the VDM-F approach is quasi static, the number of sensors has to be equal to the number of potentially modified locations for the sake of uniqueness of solution. The preceding statement is valid only when one frequency of excitation is used - for more frequencies, the number of sensors can be proportionally reduced (at least formally). Hence, if the whole structure is to be inspected for a given frequency, strain in every member must be measured. This is the major drawback of the approach.

Natural constraints are imposed on the modification coefficient $\mu_{i}$, which is nonnegative by definition (cf. (16)):

$$
\mu_{i} \geq 0
$$

If degradation of a member is considered, another constraint has to be imposed on $\mu_{i}$ :

$$
\mu_{i} \leq 1
$$

Using (8), (19), (25), the gradient of the objective function (49) with respect to the optimization variable $\mu_{i}$ is expressed as:

$$
\nabla F_{i}=\frac{\partial F}{\partial \mu_{i}}=\frac{\partial F}{\partial \varepsilon_{k}}\left(\frac{\partial \varepsilon_{k}}{\partial \varepsilon_{j}^{0}} \frac{\partial \varepsilon_{j}^{0}}{\partial \mu_{i}}+\frac{\partial \varepsilon_{k}}{\partial f_{L}^{0}} \frac{\partial f_{L}^{0}}{\partial \mu_{i}}\right)=\sum_{n_{\omega}} \frac{2}{\left(\varepsilon_{k}^{\mathrm{M}}\right)^{2}}\left(\varepsilon_{k}-\varepsilon_{k}^{\mathrm{M}}\right)\left(D_{k j}^{\varepsilon} \frac{\partial \varepsilon_{j}^{0}}{\partial \mu_{i}}+D_{k L}^{\mathrm{f}} \frac{\partial f_{L}^{0}}{\partial \mu_{i}}\right)
$$

The partial derivatives $\frac{\partial \varepsilon_{j}^{0}}{\partial \mu_{i}}$ and $\frac{\partial f_{L}^{0}}{\partial \mu_{i}}$ can be easily calculated by differentiating relation (48) with respect to $\mu_{i}$, performed $n_{\omega}$ times, independently for each considered frequency $\omega$ :

$$
\left[\begin{array}{cc}
\delta_{i j}-\left(1-\mu_{\underline{i}}\right) D_{\underline{i j}}^{\varepsilon} & -\left(1-\mu_{\underline{i}}\right) D_{\underline{i} L}^{\mathrm{f}} \\
-\omega^{2} \Delta M_{K M} B_{M j}^{\varepsilon} & \delta_{K L}-\omega^{2} \Delta M_{K M} B_{M L}^{\mathrm{f}}
\end{array}\right]\left[\begin{array}{l}
\frac{\partial \varepsilon_{j}^{0}}{\partial \mu_{i}} \\
\frac{\partial f_{L}^{0}}{\partial \mu_{i}}
\end{array}\right]=\left[\begin{array}{c}
-\varepsilon_{i} \\
\omega^{2} M_{K M} u_{M}
\end{array}\right]
$$


Note that the left-hand side matrices in (48) and (53) are alike, which simplifies computations. Only the right-hand sides vary. The optimization variable $\mu_{i}$ is updated according to the steepest descent method:

$$
\mu_{i}^{(n+1)}=\mu_{i}^{(n)}-\alpha F^{(n)} \frac{\nabla F_{i}^{(n)}}{\left[\nabla F_{i}^{(n)}\right]^{T} \nabla F_{i}^{(n)}}
$$

Superscript $(n)$ denotes values in current iteration and $(n+1)$ in subsequent iteration. The constant $\alpha$ varies in the range $0.1 \div 0.3$.

\subsection{Numerical algorithm}

Numerical algorithm solving the quasi-static inverse problem of parameter identification for harmonic excitation performs the following steps:

A. Initial calculations

(1) calculate response $\varepsilon_{k}^{\mathrm{L}}$ of the intact structure subjected successively to $n_{\omega}$ harmonic excitations of different frequencies, using a numerical model

(2) determine measured response $\varepsilon_{k}^{\mathrm{M}}$ of the structure with introduced modifications using $k$ sensors in experiment (alternatively, simulate the measured response numerically)

(3) compute influence matrices $D_{i j}^{\varepsilon}, B_{L j}^{\varepsilon}, D_{i L}^{\mathrm{f}}, D_{M L}^{\mathrm{f}}$ for $n_{\omega}$ frequencies of excitation

(4) set initial value of optimization variable to unity $\mu_{i}=1$, which implies $\Delta M_{K L}=0$, $\varepsilon_{i}^{0}=0, f_{L}^{0}=0, \varepsilon_{k}=\varepsilon_{k}^{\mathrm{L}}, u_{L}=u_{L}^{\mathrm{L}}$.

B. Iterative calculations

(1) store current value of the objective function (49) as former value $F_{\text {for }}$

(2) solve the set (53) for partial derivatives $n_{\omega}$ times

(3) calculate the gradient $\nabla F_{i}$ using (52)

(4) determine next value of the variable $\mu_{i}^{(n+1)}$ using (54)

(5) update $\Delta M_{K L}$ using (31) or (38)

(6) solve for distortions $\varepsilon_{i}^{0}, f_{L}^{0}$ using (48) $n_{\omega}$ times

(7) update $\varepsilon_{i}, u_{L}$ using (7) and (8) $n_{\omega}$ times

(8) calculate current value of the objective function $F_{\text {cur }}$

(9) check termination criterion - if $\frac{F_{\text {cur }}}{F_{\text {for }}} \leq 10^{-3}$ then STOP else go to (B1).

Note that the above algorithm can be first used at the stage of numerical model calibration, when both the measured $\varepsilon_{k}^{\mathrm{M}}$ and calculated $\varepsilon_{k}^{\mathrm{L}}$ responses refer to the intact structure. Subsequently, the same algorithm can be applied for identification of stiffness/mass modifications introduced to the structure. Then the measured response refers to the analyzed modification scenario. 


\section{Numerical examples}

\subsection{D truss structure}

As a numerical example let us consider the $2 D$ truss structure shown in Fig. 5. The initial structure consists of 20 steel elements, for which the following parameters are assumed:

- cross-section area: $A=10^{-4} \mathrm{~m}^{2}$

- Young's modulus: $E=210 \mathrm{GPa}$

- density: $\rho=7800 \frac{\mathrm{kg}}{\mathrm{m}^{3}}$

- height and width of a single section: $1 \mathrm{~m}$

- amplitude of the harmonic load: $P=200 \mathrm{~N}$

\section{Steady-state approach (VDM-F)}

In the presented frequency-domain approach, strain responses (amplitudes) are measured by sensors located in all elements using different excitation frequencies $\omega$.

The modifications of cross-section area $\mu_{i}=\frac{\hat{A}_{i}}{A_{\underline{i}}}$ in some truss elements were applied (see Fig. 5). The response of the modified structure, stored in the vector $\varepsilon_{k}^{\mathrm{M}}$, was computed numerically and then employed for the calculation of the objective function (49). The vector of the modification parameters $\mu_{i}$ was iteratively recomputed according to part (B) of the numerical algorithm. Let us follow the case 1, when only one harmonic frequency, $\omega=700 \frac{\mathrm{rad}}{\mathrm{s}}$, is used. In this case, 157 iterations had to be performed to fulfill the termination condition (B9). The identified modification parameters indicate possible defects and for some elements are under- or overestimated (see Fig. 6). For the case 2, it is assumed that the measurements are collected for 4 frequencies $\omega=[100,700,2100,3650]^{T} \frac{\mathrm{rad}}{\mathrm{s}}$ independently. Thus the dimension of the "vector" $\varepsilon_{k}^{\mathrm{M}}$ is $20 \times 4$ and for each frequency $\omega$, respective influences matrices have to be calculated. This time, the sufficient number of iterations was only 51 to fulfill the termination condition. A comparison of the identified modification parameters in both cases is shown in Fig. 6. The computational time of damage identification in both cases was similar (ca. 1 min.) on a $3 \mathrm{GHz} \mathrm{PC}$.

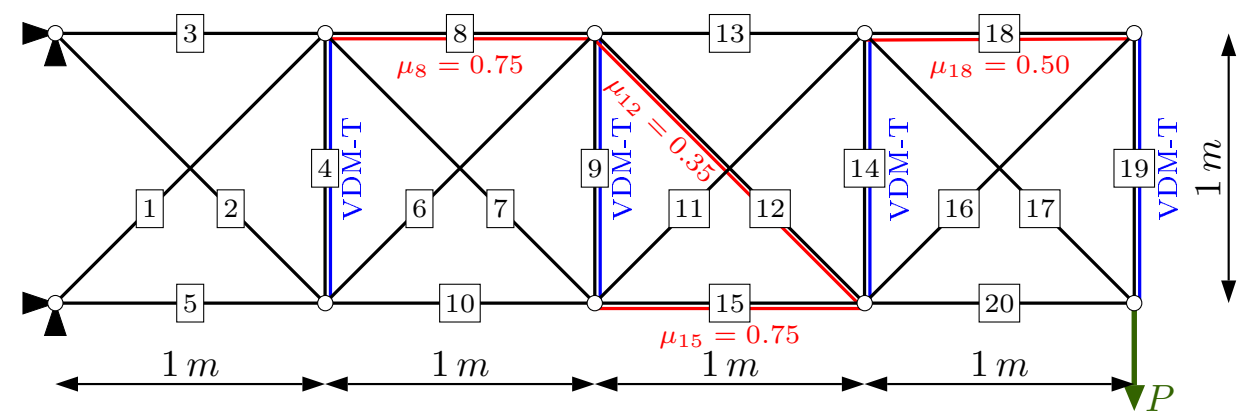

Figure 5: 2D truss structure with introduced modifications.

In practice, measured responses are accompanied by noise, thus it is advisable to include it to numerically computed responses for the modified structure. Therefore random noise affecting every strain amplitude was applied according to the following formula:

$$
\tilde{\varepsilon}_{k}^{\mathrm{M}}=\varepsilon_{\underline{k}}^{\mathrm{M}}\left(1+r_{\underline{k}}\right)
$$




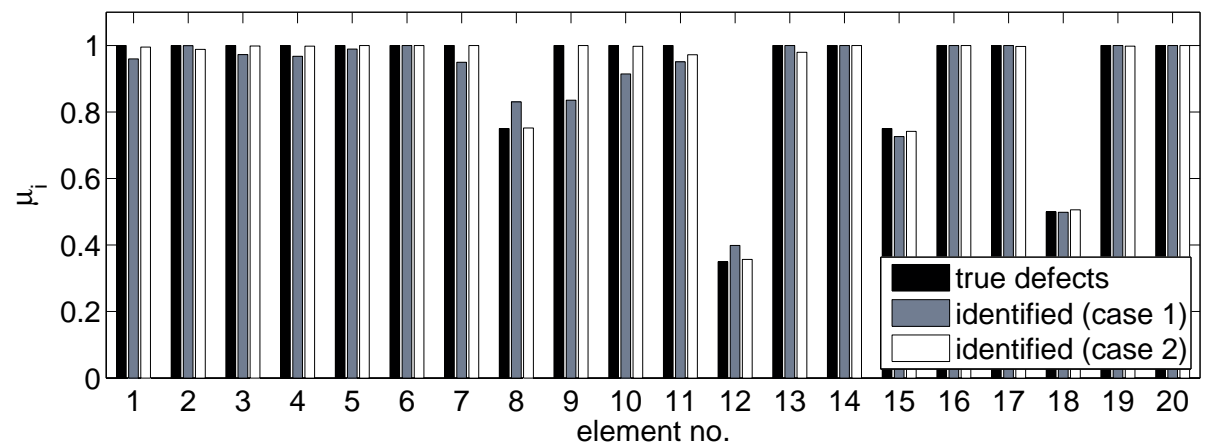

Figure 6: Identification results for 1 (case 1) and 4 (case 2) excitation frequencies.

where $r_{k}$ is an arbitrary value from the interval $\langle-0.1,0.1\rangle$. A comparison of the simulated response (for a chosen frequency) for the original and modified structure, with and without noise, is presented in Fig. 7.

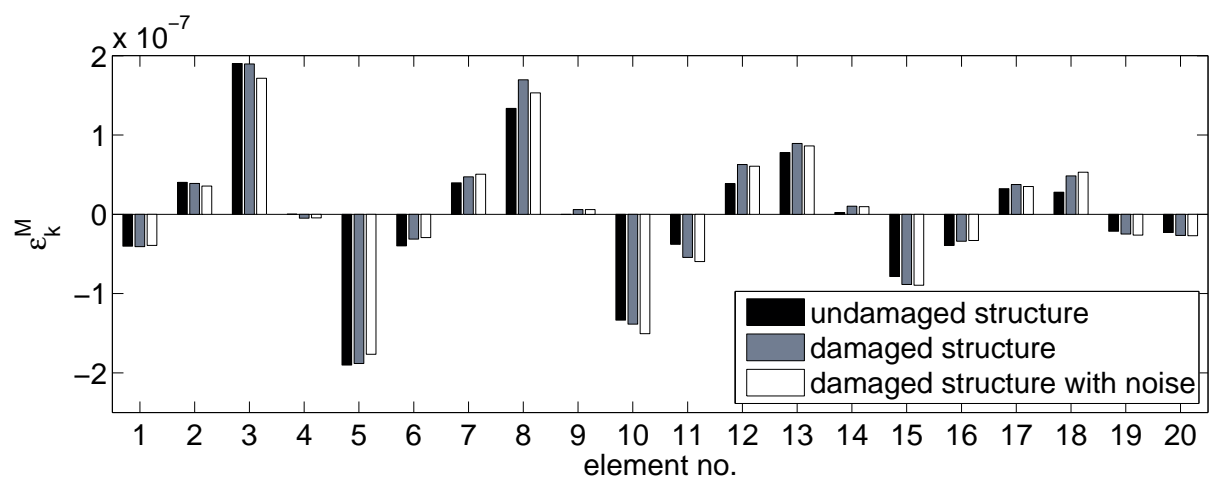

Figure 7: Influence of noise on simulated strain responses.

The described damage identification for the case 2 (cf. Fig. 5) was repeated for noisy data. The termination criterion of inverse analysis could not be met because of the noise, so the process was stopped after 300 iterations $\left(\frac{F_{\text {cur }}}{F_{\text {for }}} \approx 10^{-2}\right)$. However the obtained modification parameters distribution indicate possible defects in the structure (see Fig. 8).

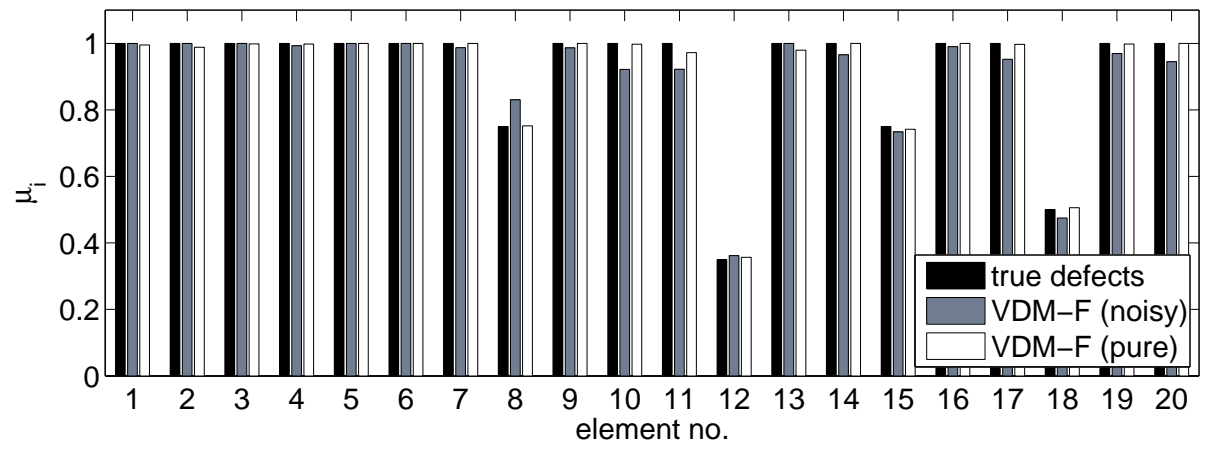

Figure 8: Identification results for pure and noisy data (case 2). 


\section{Time-domain approach (VDM-T)}

The previously presented VDM-F approach is a frequency-domain analysis based on the amplitudes responses measured in every element of the structure. On the contrary, the time-domain approach (VDM-T) allows to identify the stiffness modification parameters $\mu_{i}$ using only a few sensors collecting time-dependent responses. The same damage scenario is considered as for the VDM-F approach (cf. Fig. 5). However the stiffness modifications $\left(\mu_{i}=\frac{\hat{E}_{i}}{E_{i}}\right)$ are related to Young's modulus reductions in truss elements (not cross-section areas like in VDM-F), i.e. no mass modifications are accounted for.

In Fig. 9a the excitation signal - a windowed sine pulse with the maximum amplitude of $P=200 \mathrm{~N}$ - is depicted. Strain responses are collected from only four selected vertical elements (cf. Fig. 5). An example of strain response for the original and modified structure in element No. 1 is shown in Fig. 9b. For the other three sensing elements, the collected responses are similar. The inverse analysis using VDM-T leads to correct values, however considerable computational time is required to complete it (ca. $300 \mathrm{~min}$.). The results of damage identification for VDM-T and VDM-F are compared in Fig. 9c.

(a)

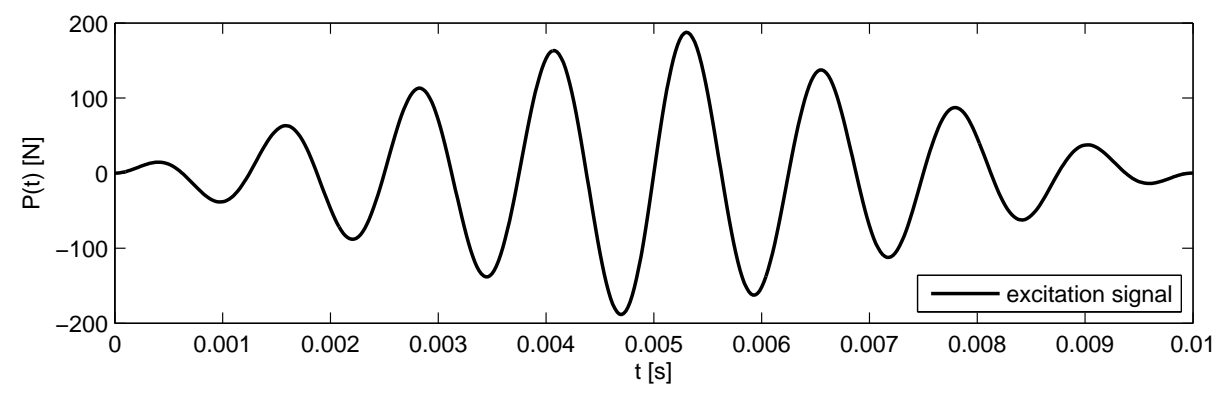

(b)

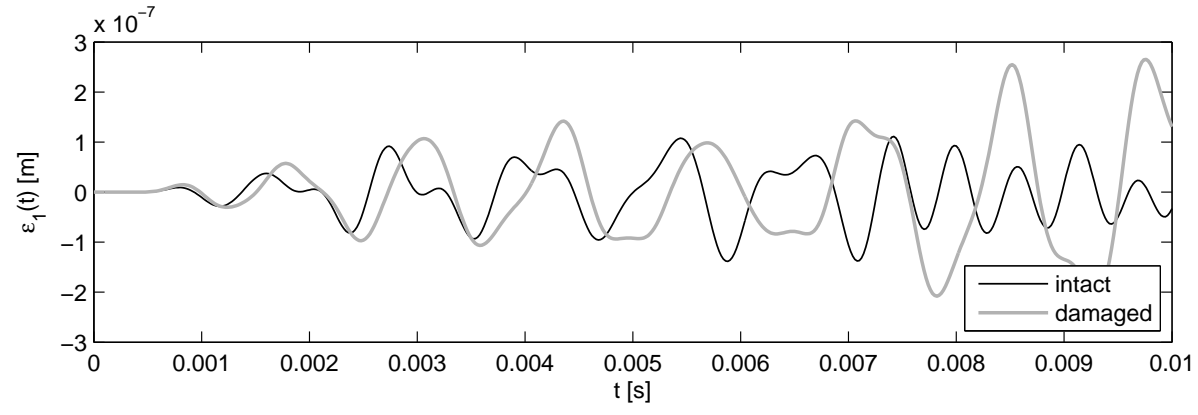

(c)

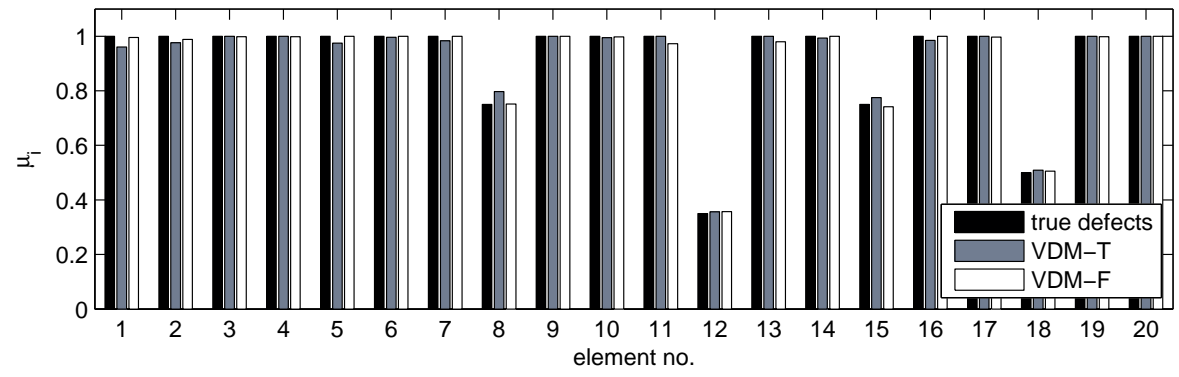

Figure 9: $2 D$ truss structure: (a) - excitation signal, (b) - strain responses in element no. 1 , (c) - results of identification via inverse analysis. 


\subsection{D beam structure}

The next numerical example is a beam structure, loaded as illustrated in Fig. (10). The investigated modification parameters are related to cross-section areas as well as moment of inertia (independently) for each finite element. Identical material and geometry parameters are assumed as in the truss example. The moment of inertia is equal to $J=2.0833 \cdot 10^{-6} \mathrm{~m}^{4}$. The structure is divided into 25 finite elements. For each one, the modification parameters $\mu_{i}^{\mathrm{A}}$ and $\mu_{i}^{\mathrm{J}}$ are evaluated. The measured strain responses (in this case axial and bending components) collected by sensors located in every finite element are used for evaluation of the modification parameters. The responses are numerically simulated for harmonic load with the amplitudes $P=100 \mathrm{~N}$ (axial force), $M=1 \mathrm{Nm}$ (bending moment) and frequency $\omega=2 \pi[10,40,155,250]^{T} \frac{\mathrm{rad}}{\mathrm{s}}$.

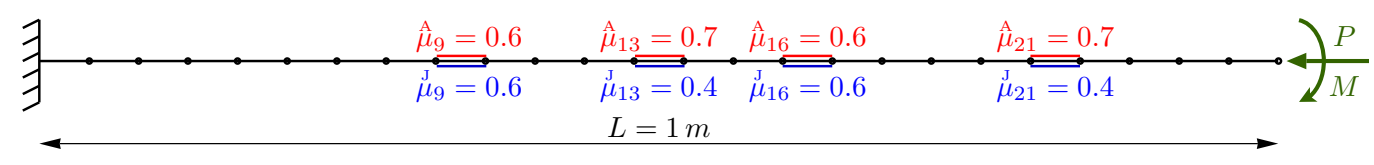

Figure 10: Beam structure with introduced modifications.

Two scenarios of damage identification (VDM-F only) were performed, namely with and without noise. Similarly to the previous example, the numerically calculated responses of the modified structure were subjected to random perturbations up to $10 \%$ according to formula (55). The results of inverse analysis are presented in Fig. 11 and Fig. 12, for both modification parameters $\mu_{i}^{\mathrm{A}}$ and $\mu_{i}^{\mathrm{J}}$, respectively. The analysis without noise was stopped when the decrease of the objective function achieved the level $10^{-4}$ (after 417 iterations). For the case with noise, the same number of iterations was performed. In both cases, the duration of inverse analysis was approximately 10 minutes on a $3 \mathrm{GHz} \mathrm{PC}$.

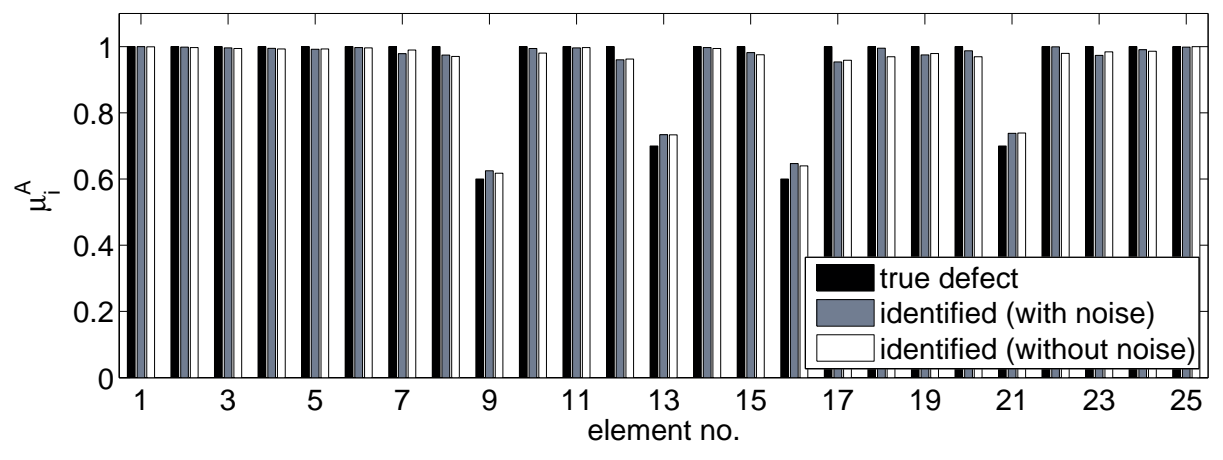

Figure 11: Identification of parameters $\mu_{i}^{\mathrm{A}}$ for pure and noisy data.

\section{Conclusions}

The paper presents an application of the Virtual Distortion Method to Structural Health Monitoring of skeletal structures (trusses and beams). Modifications of stiffness and mass are considered for simulating damage. Harmonic excitation is assumed in order to transfer the identification problem from the time domain to the frequency domain. Consequently, 


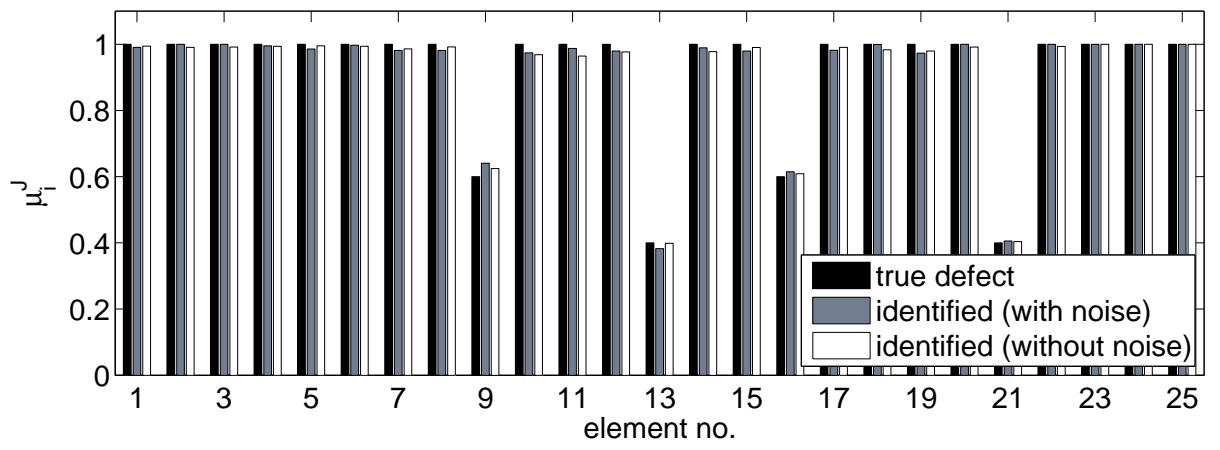

Figure 12: Identification of parameters $\mu_{i}^{\mathrm{J}}$ for pure and noisy data.

a quasi static problem is investigated with amplitudes of quantities to be analyzed only. Damping is neglected, therefore non-resonance frequencies are the subject of analysis.

Strain is the quantity contributing to the objective function. The reason is that strains vary much more smoothly in time compared to accelerations, giving reliable information even with poor sampling. On the other hand voltage proportional to strains can be measured with simple piezo-patch sensors, although there is always the matter of scaling.

The previous approach to SHM using VDM, posed in the time domain, has turned out to be quite time-consuming. The presented frequency domain approach is indeed much more efficient computationally, nevertheless it requires many more sensors to perform a successful inverse analysis. The number of sensors can be compensated with more than one excitation frequency contributing to the objective function. Also the position of actuator can be changed for a given frequency, providing more data for the identification algorithm.

The presented approach has turned out to handle noisy data quite well. The results of identification are then inferior to pure simulation, but they are still satisfactory.

Further research will be concentrated on:

- validation of the methodology by conducting a series of laboratory tests for a 3D truss structure, based on strain measurements captured by piezo-patch sensors,

- analysis of combinations of many excitation frequencies with various excitation positions to figure out optimal parameters for excitation,

- application of a more efficient optimization method (currently the steepest descent) to the identification algorithm,

- improvements to the time domain approach - extending it to handle arbitrary reallife excitations (e.g. induced by a train passing over a bridge),

- verification of the methodology in field tests

\section{Acknowledgements}

The authors would like to acknowledge the financial support from the projects R0301502 and 3T11F00930, granted by the State Committee for Scientific Research in Poland. The paper is a part of the PhD Thesis of the first author, supervised by the third author. Parts of the paper have been incorporated into the book "Smart Technologies for Safety Engineering" edited by the third author, to be published by Wiley, April 2008. 


\section{References}

[1] B. Peeters. System Identification and Damage Detection in Civil Engineering. Katholieke Univeriteit Leuven, Belgium, 2000. PhD Thesis.

[2] S. Vanlanduit, R. Pintelon, T. De Troyer, and P. Guillaume. Operational modal analysis of time varying systems using an exogenous multi-sine excitation. In Proc. of the International Conference on Noise and Vibration Engineering ISMA2006, pages 3047-3060, Leuven, Belgium, 18-20 September 2006.

[3] J. Maeck and G. De Roeck. Dynamic bending and torsion stiffness derivation from modal curvatures and torsion rates. Journal of Sound and Vibration, 225(1):153-170, 1999.

[4] C. P. Fritzen and K. Bohle. Damage identification using a modal kinetic energy criterion and "output-only" modal data - application to the Z24-brigde. In Proc. of the 2nd European Workshop on Structural Health Monitoring, pages 185-194, 7-9 July, Munich, Germany, 2004.

[5] E. J. Williams and A. Messina. Applications of the multiple damage location assurance criterion. In Proc. of the International Conference on Damage Assessment of Structures DAMAS'99, pages 256-264, Dublin, Ireland, 1999.

[6] Deraemaeker A. and Preumont A. Vibration-based damage detection using large array sensors and spatial filters. Mechanical Systems and Signal Processing, 20:16151630, 2006.

[7] J-N. Juang. Applied System Identification. Prentice Hall PTR, Englewood Cliffs, NJ, USA, 1994.

[8] B. Peeters and G. De Roeck. Reference-based stochastic subspace identification for output-only modal analysis. Mechanical Systems and Signal Processing, 13(6):855878, 1999.

[9] R. J. Allemang and D. L. Brown. A complete review of the complex mode indicator function (CMIF) with applications. In Proc. of the International Conference on Noise and Vibration Engineering ISMA2006, pages 3209-3246, Leuven, Belgium, 18-20 September 2006.

[10] B. Peeters, H. Van der Auweraer, P. Guillaume, and J. Leuridan. The PolyMAX frequency-domain method: a new standard for modal parameter estimation. Shock and Vibration, 11:395-409, 2004.

[11] W. Heylen, S. Lammens, and P. Sas. Modal Analysis Theory and Testing. Katholieke Univeriteit Leuven, Celestijnenlaan 300B, B-3001 Leuven, Belgium, 1997.

[12] U. Kirsch. A unified reanalysis approach for structural analysis, design and optimization. Structural and Multidisciplinary Optimization, 25:67-85, 2003.

[13] P. Kolakowski, M. Wiklo, and J. Holnicki-Szulc. The virtual distortion method - a versatile reanalysis tool for structures and systems. Structural and Multidisciplinary Optimization, 36(3):217-234, 2008. 
[14] P. Kolakowski, T. G. Zielinski, and J. Holnicki-Szulc. Damage identification by the dynamic Virtual Distortion Method. Journal of Intelligent Material Systems and Structures, 15(6):479-493, 2004.

[15] J. Holnicki-Szulc and J. T. Gierlinski. Structural Analysis, Design and Control by the Virtual Distortion Method. John Wiley \& Sons, Chichester, UK, 1995.

[16] M. A. Akgun, J. H. Garcelon, and R. T. Haftka. Fast exact linear and non-linear structural reanalysis and the Sherman-Morrison-Woodbury formulas. International Journal for Numerical Methods in Engineering, 50:1587-1606, 2001.

[17] EU GROWTH Project - Smart Structural Diagnostics using Piezo-Generated Elastic Waves, PIEZODIAGNOSTICS, No. GRD1-2001-40589, 2002-2005. 